\title{
Bose-Einstein condensate of kicked rotators with time-dependent interaction
}

\author{
B. Mieck ${ }^{*}$ and R. Grahant \\ Department of Physics in Essen, University Duisburg-Essen, Universitätsstrasse 5,45117 Essen, Germany
}

\begin{abstract}
A modification of the quantum kicked rotator is suggested with a time-dependent delta-kicked interaction parameter which can be realized by a pulsed turn-on of a Feshbach resonance. The mean kinetic energy increases exponentially with time in contrast to a merely diffusive or linear growth for the first few kicks for the quantum kicked rotator with a constant interaction parameter. A recursive relation is derived in a self-consistent random phase approximation which describes this superdiffusive growth of the kinetic energy and is compared with numerical simulations. Unlike in the case of the quantum rotator with constant interaction, a Lax pair is not found. In general the delta-kicked interaction is found to lead to strong chaotic behaviour.
\end{abstract}

PACS numbers: 03.75.Nt, 05.45.Pq, 02.30.Ik

The realization of Bose-Einstein condensation (BEC) of dilute gases has opened new opportunities for studying dynamical systems in the presence of many-body interactions. Previous investigations have addressed the effect of the nonlinearities due to the interactions on the dynamical localization and on the integrability of the onedimensional Gross-Pitaevskii equation (GP-equation) in the presence of a time-periodic delta-kicked external potential $v(q, t)$ [1, 2]. As is well-known the classical counterpart of the quantum kicked rotator without interaction displays chaotic motion, leading to diffusive growth in the kinetic energy above a certain value of the kick-strength $K$ of the external potential [3, 4]. In the corresponding quantum kicked rotator after a few initial kicks, during which the mean kinetic energy increases roughly linearly, the motion becomes quasiperiodic (barring special cases where quantum resonances occur) with a dynamical localization of the kinetic energy [5]-[8]. The infinite number of conserved quantities, leading to the integrability of the quantum kicked rotator, are the probabilities with which the quasi-energy states are contained in a given initial state. The inclusion of the nonlinear GP-term to the Schrödinger equation in numerical simulations was found to give rise to chaotic behaviour and delocalization of the kinetic energy [1, 2]. However, analytical considerations still show the existence of an infinite number of independent integrals of motion for the one-dimensional GP-equation with an arbitrary external potential [2, 9]. At the moment it seems difficult to reconcile this analytical result with the results of the numerical simulations.

In this paper we suggest and investigate a modification of the quantum kicked rotator by introducing a timedependent interaction $g(t)$ of delta-kicks. Furthermore, we conjecture that the numerically observed delocalization of the quantum kicked rotator with constant interaction parameter $g$ could be a consequence of employing discretizations of the nonlinear Schrödinger equation which do not preserve the property of integrability of the

\footnotetext{
*Electronic address: mieck@theo-phys.uni-essen.de
}

${ }^{\dagger}$ Electronic address: graham@uni-essen.de
GP-equation with external potential in the continuum.

The scaled GP-equation with external potential $v(q, t)=K \cos (q) \sum_{n=-\infty}^{n=\infty} \delta(t-n)$

$$
\imath \hbar \dot{\psi}=-\frac{\hbar^{2}}{2} \nabla_{q}^{2} \psi+v(q, t) \psi+g|\psi|^{2} \psi
$$

is modified by introducing a time-dependent interaction parameter $g(t)$

$$
\imath \hbar \dot{\psi}=-\frac{\hbar^{2}}{2} \nabla_{q}^{2} \psi+v(q, t) \psi+g(t)|\psi|^{2} \psi
$$

The time-dependent interaction can be achieved by a suitable time-dependent tuning to a Feshbach resonance [10]. By a short pulsed modulation of the magnetic field used for the tuning, it is possible to taylor a time-dependent coupling parameter of the form $g(t)=$ $g \sum_{n=-\infty}^{n=\infty} \delta(t-n)$ which we want to analyze here. Thus the BE-condensate, we consider in the following, receives additional delta kicks apart from that of the external potential already present for the quantum kicked rotator. In between two kicks, the BE-condensate, described by (2) follows free motion with the quantum kinetic energy, whereas in the original quantum kicked rotator with constant $g$ (Eq. (1D), the motion is determined by the integrable nonlinear Schrödinger equation (NLS) without an external potential. Therefore, numerical simulations become considerably more efficient and easier for Eq. (2) with delta-kicked interaction than for Eq. (1) because the latter equation with permanent nonlinearity needs many integration steps between two kicks [1, 2] 11].

The map for the wavefunction, stroboscopically taken after each kick $t_{n}$, with delta interaction $g(t)$ is given by

$$
\begin{aligned}
\psi\left(q, t_{n+1}^{+}\right) & =e^{-\imath / \hbar \cdot V\left(q, t_{n+1}^{-}\right)} e^{\imath \hbar / 2 \cdot \nabla_{q}^{2}} \psi\left(q, t_{n}^{+}\right) \\
V\left(q, t_{n+1}^{-}\right) & =K \cos (q)+g\left|\psi\left(q, t_{n+1}^{-}\right)\right|^{2},
\end{aligned}
$$

where the $t_{n}^{+}, t_{n}^{-}$variables refer to times immediately after $(+)$ and before (-) the occurrence of the n-th kick 12]

$$
\psi\left(q, t_{n+1}^{+}\right)=e^{-\imath / \hbar \cdot V\left(q, t_{n+1}^{-}\right)} \psi\left(q, t_{n+1}^{-}\right) .
$$


Formally, Eq. (2) with a general interaction parameter $g(t)$ can be transformed into Eq. (1) by substitution of the wavefunction $\sqrt{g(t) / g_{0}} \psi(q, t)=\widetilde{\psi}(q, t)$. In terms of the new wavefunction $\widetilde{\psi}(q, t)$, the GP-equation acquires a spatially constant, but imaginary time-dependent potential part

$$
\begin{aligned}
\imath \hbar \dot{\widetilde{\psi}} & =-\frac{\hbar^{2}}{2} \nabla_{q}^{2} \widetilde{\psi}+\widetilde{v}(q, t) \widetilde{\psi}+g_{0}|\widetilde{\psi}|^{2} \widetilde{\psi} \\
\widetilde{v}(q, t) & =v(q, t)+\imath \frac{\hbar}{2} \frac{\dot{g}(t)}{g(t)} .
\end{aligned}
$$

However, a Lax pair for the GP-equation with a complex potential $\widetilde{v}(q, t)$ and constant interaction $g_{0}$ cannot be constructed as in 2, 9] for a real external potential $v(q, t)$ :

Using the following general ansatz with $2 \times 2$ matrices for the generator $\mathcal{X}(q, t)$ and $\mathcal{T}(q, t)$ of a Lax-pair

$$
\begin{aligned}
& \mathcal{X}(q, t)=\left(\begin{array}{cc}
-\imath k_{1}(q, t) & \sqrt{g_{0}} \widetilde{\psi}^{*}(q, t) \\
\sqrt{g_{0}} \widetilde{\psi}(q, t) & \imath k_{2}(q, t)
\end{array}\right) \\
& \mathcal{T}(q, t)=\left(\begin{array}{cc}
A(q, t) & B(q, t) \\
C(q, t) & D(q, t)
\end{array}\right),
\end{aligned}
$$

with yet undetermined complex functions $A, B, C, D$ and $k_{1}, k_{2}$, the compatibility of the pair of equations

$$
\hbar \frac{\partial w}{\partial q}=\mathcal{X} w \quad \hbar \frac{\partial w}{\partial t}=\mathcal{T} w
$$

requires the following equations to be satisfied

$$
\begin{aligned}
\imath \hbar \dot{\tilde{\psi}} & =\imath \frac{\hbar}{\sqrt{g_{0}}} C_{q}+\imath(D-A) \widetilde{\psi}+\frac{\left(k_{1}+k_{2}\right)}{\sqrt{g_{0}}} C \\
-\imath \hbar \dot{\tilde{\psi}}^{*} & =-\imath \frac{\hbar}{\sqrt{g_{0}}} B_{q}+\imath(D-A) \widetilde{\psi}^{*}+\frac{\left(k_{1}+k_{2}\right)}{\sqrt{g_{0}}} \not \\
\imath \hbar \dot{k}_{1} & =-\hbar A_{q}+\sqrt{g_{0}}\left(C \widetilde{\psi}^{*}-B \widetilde{\psi}\right) \\
\imath \hbar \dot{k}_{2} & =\hbar D_{q}+\sqrt{g_{0}}\left(C \widetilde{\psi}^{*}-B \widetilde{\psi}\right) .
\end{aligned}
$$

Comparing Eqs. (11) and (12) to (67) and its complex conjugate, the terms $\imath(D-A) \widetilde{\psi}$ and $\imath(D-A) \widetilde{\psi}^{*}$ in (1112) have to be identified with $\widetilde{v}(q, t) \widetilde{\psi}$ and $\widetilde{v}^{*}(q, t) \widetilde{\psi}^{*}$ and do not allow for an imaginary part of $\widetilde{v}(q, t)$. Due to the missing of a Lax-pair, complete chaotic behaviour of the GP-equation with a general time-dependent interaction $g(t)$ can be expected. This is confirmed by our numerical simulations.

It is possible to derive an approximate recursion relation for the mean kinetic energy, just taken after kick time $t_{n}^{+}$

$$
\left\langle p^{2}\left(t_{n}^{+}\right)\right\rangle=\int_{0}^{2 \pi} d q\left|\partial_{q} \psi\left(q, t_{n}^{+}\right)\right|^{2}
$$

and time $t_{n+1}^{+},\left\langle p^{2}\left(t_{n+1}^{+}\right)\right\rangle$. The derivation is mainly based on the assumption that kick-to-kick correlations between $\psi\left(q, t_{n+1}^{+}\right)$and $\psi\left(q, t_{n}^{+}\right)$can be neglected. Applying the expression $V\left(q, t_{n+1}^{-}\right)$for the external potential and interaction before the $(n+1)$-th kick (34), the averaged kinetic energy $\left\langle p^{2}\left(t_{n+1}^{+}\right)\right\rangle$can be related to the wavefunction of the previous kick

$$
\begin{aligned}
\partial_{q} \psi\left(q, t_{n+1}^{+}\right) & =-\frac{\imath}{\hbar} \partial_{q} V\left(q, t_{n+1}^{-}\right) \psi\left(q, t_{n+1}^{+}\right) \\
& +e^{-\imath / \hbar \cdot V\left(q, t_{n+1}^{-}\right)} e^{\imath \hbar / 2 \cdot \nabla_{q}^{2}} \partial_{q} \psi\left(q, t_{n}^{+}\right) \\
\left\langle p^{2}\left(t_{n+1}^{+}\right)\right\rangle & =\frac{1}{\hbar^{2}} \int_{0}^{2 \pi} d q\left(\partial_{q} V\left(q, t_{n+1}^{-}\right)\right)^{2}\left|\psi\left(q, t_{n+1}^{+}\right)\right|^{2} \\
+ & \int_{0}^{2 \pi} d q\left|e^{\imath \hbar / 2 \cdot \nabla_{q}^{2}} \partial_{q} \psi\left(q, t_{n}^{+}\right)\right|^{2} \\
- & \frac{2}{\hbar} \int_{0}^{2 \pi} d q \Im\left[\partial_{q} V\left(q, t_{n+1}^{-}\right) \psi^{*}\left(q, t_{n+1}^{+}\right)\right. \\
\times & \left.\left(e^{-\imath / \hbar \cdot V\left(q, t_{n+1}^{-}\right)} e^{\imath \hbar / 2 \cdot \nabla_{q}^{2}} \partial_{q} \psi\left(q, t_{n}^{+}\right)\right)\right]
\end{aligned}
$$

The last integral in (17) contains only a single wavefunction $\psi^{*}\left(q, t_{n+1}^{+}\right)$at time $t_{n+1}$ so that, due to the assumption of independent phases between neighbouring kicks, this term approximately vanishes. In the second term of relation (17), the spatial derivative $\partial_{q} \psi\left(q, t_{n}^{+}\right)$is transformed by a unitary operator which cancels in the integral so that the integrand becomes $\left|\partial_{q} \psi\left(q, t_{n}^{+}\right)\right|^{2}$, and in consequence the second term is $\left\langle p^{2}\left(t_{n}^{+}\right)\right\rangle$. Taking the spatial derivative $\partial_{q} V\left(q, t_{n+1}^{-}\right)$in the first term of $\left\langle p^{2}\left(t_{n+1}^{+}\right)\right\rangle$ (17)

$$
\begin{aligned}
\partial_{q} V\left(q, t_{n+1}^{-}\right) & =-K \sin (q)+g \partial_{q} \psi^{*}\left(q, t_{n+1}^{-}\right) \psi\left(q, t_{n+1}^{-}\right) \\
& +g \psi^{*}\left(q, t_{n+1}^{-}\right) \partial_{q} \psi\left(q, t_{n+1}^{-}\right),
\end{aligned}
$$

we only keep expressions with absolute values of wavefunctions and their derivatives and obtain

$$
\begin{aligned}
& \hbar^{2}\left\langle p^{2}\left(t_{n+1}^{+}\right)\right\rangle \approx K^{2} \int_{0}^{2 \pi} d q \sin ^{2}(q) \underbrace{\left|\psi\left(q, t_{n+1}^{+}\right)\right|^{2}}_{1 /(2 \pi)} \\
& +2 g^{2} \int_{0}^{2 \pi} d q\left|\partial_{q} \psi\left(q, t_{n+1}^{-}\right)\right|^{2} \underbrace{\left|\psi\left(q, t_{n+1}^{-}\right)\right|^{2}}_{1 /(2 \pi)} \underbrace{\left|\psi\left(q, t_{n+1}^{+}\right)\right|^{2}}_{1 /(2 \pi)} \\
& +\hbar^{2}\left\langle p^{2}\left(t_{n}^{+}\right)\right\rangle,
\end{aligned}
$$

where the absolute values of $\psi\left(q, t_{n+1}^{-}\right)$and $\psi\left(q, t_{n+1}^{+}\right)$ are replaced by the mean density $1 /(2 \pi)$. Since the wavefunction $\psi\left(q, t_{n+1}^{-}\right)$differs from $\psi\left(q, t_{n}^{+}\right)$only by the time development with $\exp \left\{\imath \hbar / 2 \cdot \nabla_{q}^{2}\right\}$, the average of $\left|\partial_{q} \psi\left(q, t_{n+1}^{-}\right)\right|^{2}$ can be replaced by $\left|\partial_{q} \psi\left(q, t_{n}^{+}\right)\right|^{2}$.

This results in a recursive relation between $\left\langle p^{2}\left(t_{n+1}^{+}\right)\right\rangle$ and $\left\langle p^{2}\left(t_{n}^{+}\right)\right\rangle$

$$
\left\langle p^{2}\left(t_{n+1}^{+}\right)\right\rangle=\frac{K^{2}}{2 \hbar^{2}}+\left(1+\frac{g^{2}}{2 \pi^{2} \hbar^{2}}\right)\left\langle p^{2}\left(t_{n}^{+}\right)\right\rangle .
$$

In the case of vanishing interaction parameter $g,\left\langle p^{2}\left(t_{n}^{+}\right)\right\rangle$ increases linearly with $K^{2} /\left(2 \hbar^{2}\right)$, as expected for the initial classical diffusive regime of the original quantum 
kicked rotator. However, the phenomenon of quantum localization cannot be derived from the recursive relation which assumes no kick-to-kick correlation of the phases of the wavefunctions at time $t_{n}$ and $t_{n+1}$ and neglects the correlations due to the presence of quasi-energy eigenstates. For finite interaction $g$ quasi-energy states no longer exist and the assumption leading to (20) can be consistent. Indeed, due to Eq. (20), the mean kinetic energy follows an exponential or superdiffusive growth, indicating strong chaotic behaviour, which makes the assumption of statistical independence of subsequent kicks self-consistent. The continuum limit of the recursion relation (20) also yields an exponential growth of $\left\langle p^{2}(t)\right\rangle$

$$
\left\langle p^{2}(t)\right\rangle=\frac{\pi^{2} K^{2}}{g^{2}}\left[\exp \left(\frac{g^{2}}{2 \hbar^{2} \pi^{2}} t\right)-1\right]
$$

where $\left\langle p^{2}(t=0)\right\rangle$ vanishes if the initial wavefunction $\psi(q, t=0)=1 / \sqrt{2 \pi}$ is taken with zero momentum as in our simulations. Relation (21) for $\left\langle p^{2}(t)\right\rangle$ interpolates between purely linear increase for small $g$ and exponential growth for strong interactions.

The comparison of the recursive relation for $\left\langle p^{2}\left(t_{n}^{+}\right)\right\rangle$ (20) and $\left\langle p^{2}(t)\right\rangle$ (21) with numerical simulations yields a qualitative agreement on a logarithmic scale (see Figs. 1 to [3). In Fig. 33 we display on a logarithmic scale the mean kinetic energy for the delta-kick interaction $g(t)=g \sum_{n=-\infty}^{n=\infty} \delta(t-n)$ with $g=5$ and for the external potential $v(q, t)=K \cos (q) \sum_{n=-\infty}^{n=\infty} \delta(t-n)$ with kick strength $K=1$. Our estimate (20) and (21) for $\left\langle p^{2}\left(t_{n}^{+}\right)\right\rangle$ gives a consistent value for the slope whereas the absolute values of $\left\langle p^{2}\left(t_{n}^{+}\right)\right\rangle$differ by a constant factor from the numerical results. This deviation may result from the substitution of the average of the product of the densities with the product of the average of the wavefunctions (cf. Eq. (19)). The phenomenon of quantum localization as for vanishing interaction $g=0$ or small but constant $g$ has not been obtained in our simulations with available number of space points up to $2^{16}$ on the periodic interval $[0,2 \pi)$. The increase of the mean kinetic energy only ended at the maximum momentum squared which is limited by the spatial intervals. The complete missing of any sign of quantum localization and the superdiffusive increase of $\left\langle p^{2}(t)\right\rangle$ are the main differences to the original quantum kicked rotator. In the case of constant interaction parameter $g(t)=g$ (Eq. (1)), a slow increase of the mean kinetic energy can be observed in numerical simulations 1, 2]. We conjecture that that this delocalization may be caused by a nonintegrable discretization. According to Ref. 9] and our considerations, the obvious discrete version of the GP-equation (11) is not integrable $\left(\psi_{n}(t)=\psi\left(q_{n}, t\right)\right)$

$\imath \hbar \dot{\psi}_{n}=-\frac{\hbar^{2}}{2} \frac{\psi_{n+1}-2 \psi_{n}+\psi_{n-1}}{(\Delta q)^{2}}+v_{n}(t) \psi_{n}+g\left|\psi_{n}\right|^{2} \psi_{n}$

and does not possess a Lax-pair, whereas the following discrete Schrödinger equation with constant interaction parameter $g$ can be derived from a compatibility condition with appropriate generators $\mathcal{X}$ and $\mathcal{T}$ for a Lax-pair

$$
\begin{aligned}
& \imath \hbar \dot{\psi}_{n}=-\frac{\hbar^{2}}{2} \frac{\psi_{n+1}-2 \psi_{n}+\psi_{n-1}}{(\Delta q)^{2}} \\
& +\frac{1}{2}\left(v_{n}(t)+v_{n+1}(t)\right) \psi_{n}+\frac{g}{2}\left|\psi_{n}\right|^{2}\left(\psi_{n+1}+\psi_{n-1}\right) .
\end{aligned}
$$

The mean kinetic energy derived from (23) and (22) can therefore be expected to behave quite differently, but (23) is, unfortunately, more difficult to simulate accurately than (22), and has not been analyzed so far.

Discrete versions of differential equations need not have in general the same integrability or chaoticity properties as their continuous counterparts [9]. However, the modification of the quantum kicked rotator by a deltakicked interaction, we have studied here, is free from such difficulties since it gives a unique map from the continuum equation (2) to the time development (3) of the wavefunction with only free motion between two kicks. The superdiffusive growth and strong delocalization of the mean kinetic energy should be observable experimentally by a suitable tuning to a Feshbach resonance. A realization of a ring structure for a BE-condensate, on which short potential kicks are applied, has been suggested 2]. Qualitatively, a stronger expansion rate of the condensate should be observed for the delta-kicked interaction because of the exponential increase of the mean kinetic energy.

\section{Acknowledgments}

This work was supported by the Deutsche Forschungsgemeinschaft within the SFB/TR 12 . We gratefully acknowledge the discussion with L. Santos who suggested the use of a delta-kicked interaction.

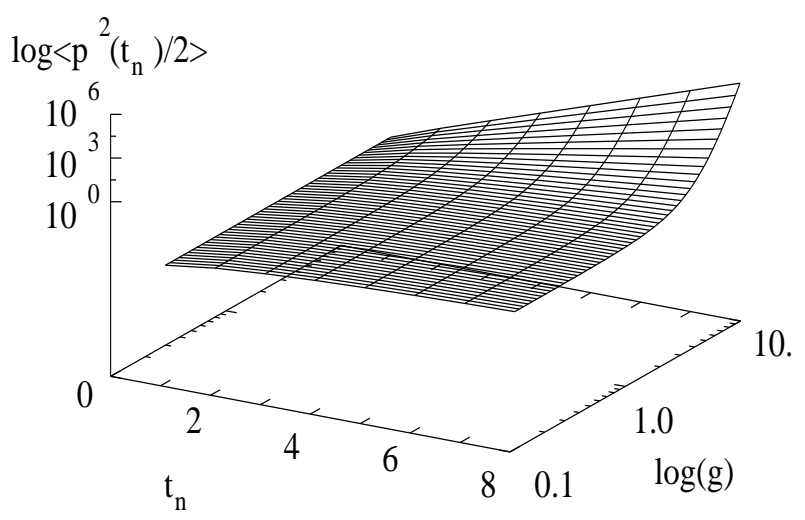

FIG. 1: Logarithm of the mean kinetic energy determined from the recursive relation (20) for vanishing initial momentum of the wavefunction $\psi\left(q, t_{0}\right)=1 / \sqrt{2 \pi}$ with a kick strength of the external potential of $K=1.0 . \log \left\langle p^{2}\left(t_{n}\right) / 2\right\rangle$ is displayed for the first eight kicks $t_{n}$ versus the logarithm of the parameter $g$ of the delta-kicked interaction, ranging from $g=0.1$ to $g=10.0$. 


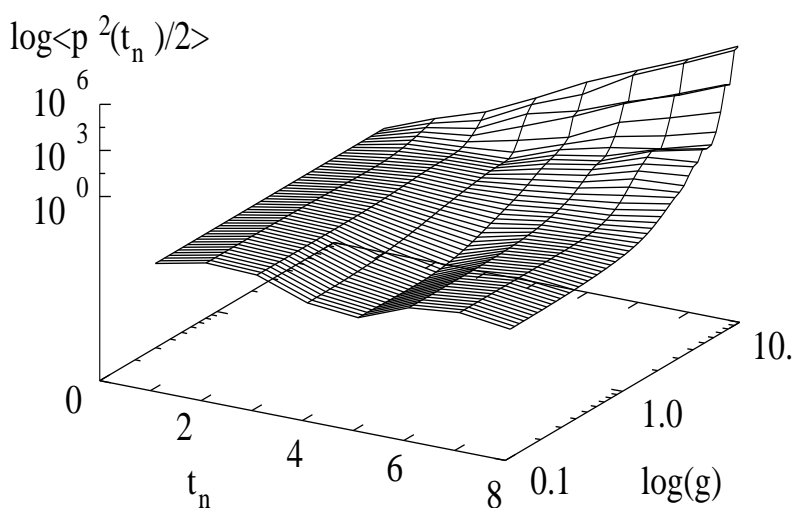

FIG. 2: Results of the numerically obtained averaged kinetic energy on a logarithmic scale for the same parameters as in Fig. 1] (Configurations of the axes are the same as in Fig. 11) Qualitative agreement for the superdiffusive growth of $\left\langle p^{2}\left(t_{n}\right) / 2\right\rangle$ is obtained with the exponential relation (20).

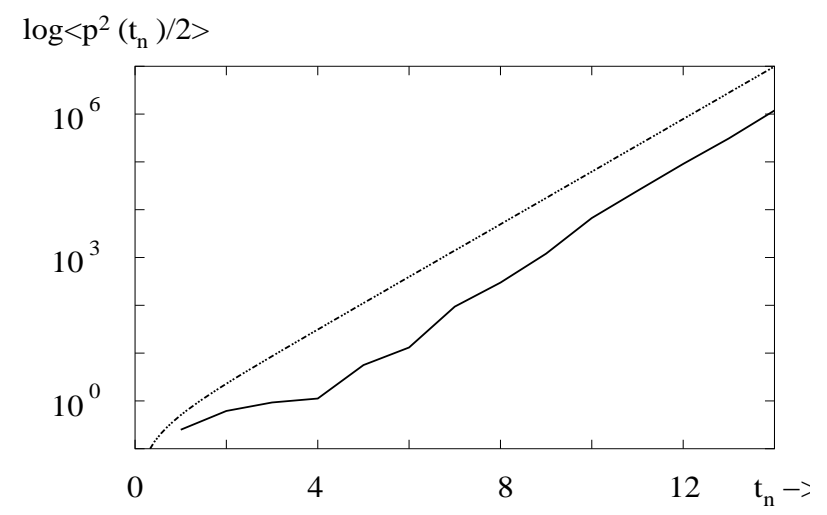

FIG. 3: Comparison of the exponential relation (21) (dashdotted upper line) for $g=5.0$ of the delta-kicked interaction and the kick strength $K=1.0$ with the corresponding numerical simulations (lower solid line). The slope of the exponential increase of the kinetic energy, obtained by (21), is in good agreement with the computations whereas absolute values differ by a constant factor.

[1] Ch.Zhang, J.Liu, M.G.Raizen, and Q.Niu, Phys. Rev. Lett 92, 054101 (2004)

[2] B. Mieck and R. Graham, J. Phys. A 37 L581-L588

[3] B.V.Chirikov, Phys. Reports 52, 265 (1979)

[4] J.M.Greene, J. Math. Phys. 20, 1183 (1979)

[5] G. Casati, B.v.Chirikov, J.Ford, and F.M.Izrailev, in 'Lecture Notes in Physics 93', G. Casati and J.Ford eds., Springer, Berlin 1979; B.V.Chirikov, F.M.Izrailev, and D.L.Shepelyansky, Sov. Sci. Rev. 2C, 209 (1981)

[6] D.R.Grempel, R.E.Prange, and S.Fishman, Phys. Rev. A 29, 1639 (1984)

[7] R.Graham, M.Schlautmann, and P.Zoller, Phys. Rev. A45, R19 (1992)

[8] F.L.Moore, J.C.Robinson, C.Bharucha, P.E.Williams, and M.G.Raizen, Phys. Rev. Lett. 73, 2974 (1994); F.L.Moore, J.C.Robinson, C.F.Bharucha, B.Sundaram, and M.G.Raizen, Phys. Rev. Lett. 75, 4598 (1995)

[9] M.J.Ablowitz, B.Prinari, and A.D.Trubatch, 'Discrete and Continuous Nonlinear Schroedinger Systems', London Math. Soc. Lecture Note Series 302, Cambridge University Press, Cambridge 2004

[10] M. Olshanii, Phys. Rev. Lett. 81, 938 (1998)

[11] We are grateful to L. Santos, who suggested this idea to us in a private discussion.

[12] We assume here that the two types of kicks (of the potential and of the interaction) occur at the same time, but this is of no importance for our conclusions. 